\title{
Investigations on Combining Ability and Heterosis in Hybrid Rice
}

\author{
R. Jayalakshmi ${ }^{*}$, R. Saraswathi ${ }^{2}$ and K. Amudha ${ }^{2}$ \\ ${ }^{1}$ Centre for Plant Breeding and Genetics, ${ }^{2}$ Department of Rice, Centre for Plant Breeding and \\ Genetics, Tamil Nadu Agricultural University, Coimbatore, Tamil Nadu, India \\ *Corresponding author
}

\section{A B S T R A C T}

\begin{tabular}{l} 
Ke y w o r d s \\
Hybrid rice, \\
Combining ability, \\
Gene action, \\
Heterosis \\
Article Info \\
$\begin{array}{l}\text { Accepted: } \\
\text { 20 January } 2021 \\
\text { Available Online: } \\
\text { 10 February } 2021\end{array}$ \\
\hline
\end{tabular}

Keywords

Hybrid rice, Combining ability, Gene action, Article Info

Accepted: Available Online: 10 February 2021
Combining ability analysis and heterosis study was made in forty-two rice hybrids developed by crossing fourteen lines with three testers and resultant evaluation with six checks of different duration groups. The sca variance was greater than gca variance for all the traits under study indicating the presence of non-additive gene action. Among parents, lines 1927, 1877 and tester COMS 24A were discovered as the best general combiners for single plant yield. Eight hybrid combinations viz., COMS 23A $\times 1933 / 1$, COMS $24 \mathrm{~A} \times$ 1912/2, COMS 24A× 1927, COMS 24A × 1969/1, COMS 24A $\times 2007$, COMS 24A $\times$ 1877 , COMS $25 \mathrm{~A} \times 1967$ and COMS $25 \mathrm{~A} \times 1726$ had significant mean, positive $s c a$ effects and standard heterosis over their respective checks. All the hybrids had good flowering synchrony between respective parents and may be employed in the upcoming hybrid rice breeding programmes for test verification and exploitation.

\section{Introduction}

Rice is the pivotal food crop feeding over half of the world's population. Rice occupies the second largest area next to wheat. Rice is the core ingredient of Asia's food platter. Asia is the largest producer of paddy and $90.7 \%$ of world paddy production is from Asia (FAO STAT 2018-2019). Majority of the farming community have paddy cultivation as their sole source of income. So, enhancing the productivity of paddy is a never-ending process besides considering profitable and sustainable farm practices. The present challenge in paddy cultivation is to feed the world's humongous population with limited resources. Hybrid breeding that combines superior alleles from both parents to synthesize a better $F_{1}$ that is heterotic over its inbred parents and ruling inbred varieties is most efficient and fastest approaches in rice breeding to increase the rice production. Progress and success of hybrid rice technology in China (Cheng et al., 2007), proved that it is a practically feasible solution to overcome the yield plateau witnessed in rice production. Chinese scientists exploited cytoplasmic male sterility restorer system for exploiting hybrid vigour to increase the production (Hwa and Yang, 2008). This system bridges the yield gap by elevating the rice production and makes sustainable utilization of environment. Expression of hybrid vigour (heterosis) has led to increase in 
the yield potential of hybrid rice varieties by 15 to $20 \%$ over current commercial cultivars (Virmani and Edwards, 1983).

In India the area under hybrid rice is $3.0 \mathrm{~m}$. ha and more than $80 \%$ of the total hybrid rice area is in the states of Uttar Pradesh, Jharkhand, Chhattisgarh, Madhya Pradesh, Odisha and Haryana. Hybrid rice research is undertaken over the last three decades and 129 hybrids have been released upto 2019-20, of which 40 are state releases (AICRIP Progress Report, 2019). Five hybrids viz., ADTRH 1, CORH 1 to CORH 3 and Hybrid $\mathrm{CO} 4$ have been released by Tamil Nadu Agricultural University and the efforts are being continued to identify new heterotic combinations.

The first step in any hybrid programme is assessing the parental lines for their gene action and combining ability so that heterosis can be directly exploited at its best to get superior hybrids. The current experiment was carried out with the objective of identifying superior hybrids and to evaluate the combining ability of three stable cytoplasmic genic male sterile lines and 14 identified restorer lines with the aim of exploiting heterosis in rice hybrids for yield and yield attributing traits.

\section{Materials and Methods}

The research was undertaken at the Department of Rice, Centre of Plant Breeding and Genetics (CPBG), Tamil Nadu Agricultural University, Coimbatore. Three male sterile lines viz., COMS 23A, COMS 24A and COMS 25A having Wild Abortive cytoplasm (WA) mated with fourteen genetically diverse potential restorer lines in Line $\times$ Tester mating design and their corresponding $F_{1}$ hybrids constituted the material of study. The crossing programme was carried out during Kharif 2019 and hybrids were assessed along with six checks during Rabi 2019.

A spacing of $30 \mathrm{~cm} \times 20 \mathrm{~cm}$ was followed to transplant thirty days old seedlings in a row length of $4.1 \mathrm{~m}$ replicated thrice in a randomized block design. Required package of practices to raise a healthy crop was adhered. From each replication, five plants were randomly selected and biometrical observations viz., plant height $(\mathrm{cm})$, number of productive tillers per plant, panicle length $(\mathrm{cm})$, panicle weight $(\mathrm{g})$, number of filled grains per panicle, number of primary branches per panicle, number of secondary branches per panicle, 100 seed weight $(\mathrm{g})$, spikelet fertility (\%) and single plant yield was recorded. Also, days to $50 \%$ flowering for a single row was observed. Mean data was subjected to statistical analysis. Analysis of variance and Line $\times$ Tester analysis was done according to protocol given by Panse and Sukhatme (1985) and (Kempthorne, 1957) respectively.

\section{Results and Discussion}

Combining ability analysis gives us information on nicking ability among parents and to realize the nature and magnitude of gene action present. The analysis of variance for combining ability for different biometrical traits is presented in Table 1. The results indicated the presence of significant differences for crosses and line $\times$ tester effect for all the eleven characters under study. Except spikelet fertility, rest of the characters exhibited significant line effect while the tester effect was significant for all the parameters except plant height. Significant differences among treatments display the variation in the genetic material used. Singh et al., (2019) also reported significant linex tester effect for yield and yield component traits. Osama et al., (2017) reported significant effect for cross, lines, testers and 
line $\times$ tester effect for panicle length, panicle weight, number of filled grains, spikelet fertility and yield. Gramaje et al., (2016) obtained significant differences among 11 restorers, three CMS lines and their 33 hybrids in days to maturity, plant height, productive tillers, panicle length, filled grains per panicle, total spikelet count, spikelet fertility, 1000 grain weight, grain length and width, and grain yield. The ratio of gca to sca was less than unity for all the traits under study. From this we can conclude the preponderance of non-additive gene action over additive gene action. Results infer the control of dominance and epistatic gene interactions over the traits. These interactions can be effectively utilized in heterosis breeding. Tiwari et al., (2011), Parimala et al., (2018) and Singh et al., (2019) also observed non additive gene action for yield and yield attributing traits.

Relative contribution of lines, testers and lines $x$ testers interaction to the total variance is given in Table 2. The contribution of lines towards total variance was higher for the traits viz., days to $50 \%$ flowering, number of productive tillers per plant, panicle length, number of primary and secondary branches and 100 seed weight while for rest of the traits, lines $\times$ testers interaction contributed more to the total variance when compared to testers. Devi and Lal (2015)and Hasan et al., (2015) also recorded greater contribution from lines and lines $\times$ tester interaction for major biometrical traits.

From the perusal of the per se performance of parents presented in Table 3, the following conclusions were made i.e., lines 1881,1871, 1729 and 1912/2 exhibited significant mean values for days to $50 \%$ flowering (101-105 days). Line 1969/1 displayed significant low mean value of $85 \mathrm{~cm}$ for plant height. For number of productive tillers per plant, lines 1927 (26.33 nos.) and 1747 (24.6 nos.) were highly significant. For single plant yield the lines 1747 (45.21g), 1734 (40.39) and 1877 $(38.48 \mathrm{~g})$ were highly significant. These lines had highly significant values for 100 seed weight also. In addition, two lines 1726and 1729 were also highly significant for 100 seed weight. Line 1877 had significant mean values for panicle length $(28.13 \mathrm{~cm})$, panicle weight $(5.07 \mathrm{~g})$, number of secondary branches (55.13) and number of filled grains (188.44). Tester COMS 23A had significant mean value for days to $50 \%$ flowering (101 days) whereas COMS 25A expressed significance for number of productive tillers per plant (23.20), number of filled grains per panicle (191.89) and 100 seed weight (1.76g).

The average performance of a line in a series of hybrid combinations known as general combining ability is an indication of superiority of parents. The gca effects of parents are depicted in Table 4. Significant effects in the negative direction are desirable for the traits days to $50 \%$ flowering and plant height. The lines namely 1877, 1871, 1881, 1729 and 1933/1 and the tester COMS 23A had negative gca effects for days to $50 \%$ flowering. For plant height, the lines 1729, 1734 and 1871 had negative gca effects. Parimala et al., (2018)had crossed five CMS lines with twelve restorer lines to generate sixty hybrids and also reported significant negative gca effects for days to $50 \%$ flowering and plant height among parents.

For characters panicle length, panicle weight, number of primary branches number of secondary branches, number of filled grains per panicle and single plant yield, the line 1927 had highly significant gca effects. Line 1877 displayed significant gca effects in desirable direction for days to $50 \%$ flowering, panicle length, panicle weight, spikelet fertility, 100 seed weight and single plant yield. Lines 1726, 1734, 1877, 1933/1, 1927, 1967 and 1969/1 exhibited positive significant 
gca effects for single plant yield. Lines with significant positive gca effects for 100 seed weight were 1729, 1734, 1747 and 1726. Tester COMS 24A exhibited highly significant $g c a$ effects for number of secondary branches per panicle, spikelet fertility, 100 seed weight and single plant yield. For number of productive tillers per plant, testers COMS 24A and COMS 25A had positive significant $g c a$ effects. On the whole, the lines 1877 and 1927 were best combiners as they had significant gca effect in desirable direction for six biometrical traits each. Tester COMS 24A emerged as the best general combiner among testers as it showed significant gca effects for five traits. Hence, all these above lines and testers can be concluded as good general combiners for the traits studied. The current experiment indicated the presence of good combiners among parents. On a similar note, Thippeswamy et al., (2016) had identified six restorer lines and three CMS line as good combiners among eighteen parents.

The per se performance for 42 hybrids for all the 11 traits is given in Table 5. Among 42 hybrids, ten hybrids had significantly lower mean values (85.67 to 97.33 days) for days to $50 \%$ flowering indicating earliness in the hybrids. Seven hybrids had significant mean values for number of productive tillers $(26.47$ to 36.07 nos.). Fifteen hybrids had significant mean values for number of filled grains per panicle (163.33 to 215.20 nos.). For 100 seed weight, nine cross combinations (2.04 to $2.36 \mathrm{~g}$ ) had significant mean value. Thirteen cross combinations (49.30 to $68.44 \mathrm{~g}$ ) had highly significant and three had significant (46.11 to $46.26 \mathrm{~g}$ ) mean values for single plant yield. Of these, the hybrids COMS 23A $\times$ 1970 and COMS24A $\times 1877$ had significant mean performance for five other yield attributing traits also in this study.

Specific combining ability effects that imply the superior or inferior performance of a cross in comparison with average performance of their parents is depicted in Table 6. From the perusal of the results, we could conclude that eleven hybrids had negative sca effects for days to $50 \%$ flowering. For plant height, the hybrid COMS 25A× 1734displayed negative sca effect.

Nine and thirteen cross combinations had positive significant sca effects for number of productive tillers and number of filled grains per panicle. Both of these characters are crucial in obtaining superior yield. Parimala et al., (2018) who had evaluated sixty hybrids $(5 \mathrm{~A} \times 12 \mathrm{R})$ have reported significant $s c a$ effects for the above-mentioned characters in desirable direction and so also, Sudeepthi et al., (2018) akin to our results. Fifteen hybrids possessed positive significant sca effect for 100 seed weight. For single plant yield, fifteen hybrids namely COMS23A × 1927, COMS 24A $\times 1881$, COMS 24A $\times 1877$, COMS $25 \mathrm{~A} \times 1747$, COMS $24 \mathrm{~A} \times 1871$, COMS 24A $\times 1969 / 1$, COMS 23A $\times 1747$, COMS 24A $\times 2007$, COMS 24A $\times 1912 / 2$, COMS 24A $\times 1927$, COMS 25A $\times 1729$, COMS 23A $\times 1970$, COMS 23A $\times 1933 / 1$, COMS 25A $\times 1967$ and COMS 25A $\times$ 1726 had positive significant sca effect. Singh et al., (2019) had developed seventy-two hybrids by crossing four CMS lines with 16 diverse restorer lines had reported twenty hybrids with positive significant sca effects. Thippeswamy et al., (2016)and Parimala et al., (2018) have also reported hybrids with positive significant sca effects. Ni et al., (2009) from the study of 20 hybrids for genetic distance and sca concluded that heterosis can be reflected by specific combining ability but sca cannot be reflected by genetic distance and that hybrid rice combinations with high special combining ability can be used to breed strong superior combinations with practical values. 
Table.1 Analysis of variance for combining ability for grain yield and contributing characters in rice

\begin{tabular}{|c|c|c|c|c|c|c|c|c|c|c|c|c|}
\hline $\begin{array}{l}\text { Source of } \\
\text { variation }\end{array}$ & df & $\begin{array}{l}\text { DFF } \\
(\%)\end{array}$ & $\begin{array}{l}\text { PH } \\
(\mathbf{c m})\end{array}$ & NPT & $\begin{array}{l}\text { PL } \\
(\mathbf{c m})\end{array}$ & $\begin{array}{c}\text { PW } \\
\text { (g) }\end{array}$ & PB & SB & NFG & SF (\%) & $\begin{array}{c}\text { 100SW } \\
\text { (g) }\end{array}$ & $\begin{array}{c}\text { SPY } \\
(\mathrm{g})\end{array}$ \\
\hline REPLICATION & 2 & 0.51 & 73.43 & 9.35 & 1.67 & 0.29 & 0.04 & 7.005 & 3.03 & 3.21 & 0.00 & 19.97 \\
\hline CROSS & 41 & $109.17^{* *}$ & $64.59^{* * *}$ & $62.17^{* *}$ & $3.64^{* *}$ & $1.58^{* *}$ & $2.05^{* *}$ & $161.42^{* * *}$ & $2639.67^{* *}$ & $12.54^{* *}$ & $0.09^{* *}$ & $310.99^{* *}$ \\
\hline LINE & 13 & $174.10^{* *}$ & $85.31^{* *}$ & $98.90^{* *}$ & $6.73^{* *}$ & $1.04^{* *}$ & $3.35^{* *}$ & $328.37^{* *}$ & $2418.80^{* * *}$ & 5.11 & $0.15^{* *}$ & $359.14^{* * *}$ \\
\hline TEST & 2 & $33.56^{* *}$ & 35.09 & $146.77^{* *}$ & $3.56^{* *}$ & $10.06^{* *}$ & $4.45^{* *}$ & $202.85^{* *}$ & $10239.13^{* * *}$ & $62.31^{* *}$ & $0.10^{* *}$ & $248.70^{* * *}$ \\
\hline LXT & 26 & $82.52^{* *}$ & $56.50^{* * *}$ & $37.29^{* *}$ & $2.11^{* * *}$ & $1.20^{* *}$ & $1.21^{*}$ & $74.77^{* *}$ & $2165.53^{* *}$ & $12.42^{* *}$ & $0.05^{* *}$ & $291.71^{* *}$ \\
\hline ERROR & 82 & 2.91 & 21.84 & 3.57 & 0.49 & 0.11 & 0.69 & 1.97 & 104.30 & 4.65 & 0.00 & 5.56 \\
\hline GCA & & 0.36 & 0.11 & 0.34 & 0.02 & 0.01 & 0.01 & 1.17 & 6.45 & 0.0016 & 0.00 & 0.26 \\
\hline SCA & & 26.54 & 11.56 & 11.24 & 0.54 & 0.36 & 0.17 & 24.26 & 687.08 & 2.59 & 0.02 & 95.38 \\
\hline GCA/SCA & & 0.01 & 0.01 & 0.03 & 0.0370 & 0.01 & 0.07 & 0.0482 & 0.01 & 0.0006 & 0.03 & 0.003 \\
\hline
\end{tabular}

*Significant at 5\%, ** Significant at 1\%

DFF- Days to 50\% flowering PW-Panicle weight SF-Spikelet fertility

PH -Plant height PB- Number of primary branches 100SW- hundred seed weight

NPT-Number of productive tillers SB- Number of secondary branches SPY-Single plant yield

PL-Panicle length NFG-Number of filled grains per panicle

Table.2 Proportional contribution of lines, testers and interaction to total variance

\begin{tabular}{|l|c|c|c|c|}
\hline \multirow{2}{*}{\multicolumn{1}{c|}{ Characters }} & \multicolumn{3}{c|}{ Proportional contribution (\%) } \\
\cline { 2 - 5 } & Lines $(\mathbf{R})$ & Testers(T) & Lines x Testers & \multicolumn{1}{c|}{ Total } \\
\hline Days to 50 \% flowering & 50.57 & 1.50 & 47.94 & 100 \\
\hline Plant height (cm) & 41.88 & 2.65 & 35.47 & 100 \\
\hline Number of productive tillers per plant & 50.44 & 11.52 & 36.04 & 100 \\
\hline Panicle length (cm) & 58.55 & 4.77 & 48.06 & 100 \\
\hline Panicle weight (g) & 20.92 & 31.03 & 37.55 & 100 \\
\hline Number of primary branches & 51.86 & 10.60 & 29.37 & 100 \\
\hline Number of secondary branches & 64.50 & 6.13 & 52.02 & 100 \\
\hline Number of filled grains per panicle & 29.05 & 18.92 & 62.83 & 100 \\
\hline Spikelet fertility (\%) & 12.93 & 24.24 & 38.82 & 100 \\
\hline 100 seed weight (g) & 55.58 & 5.60 & 59.48 & 100 \\
\hline Single plant yield (g) & 36.32 & 3.90 & & \\
\hline
\end{tabular}


Table.3 Mean performance of lines and testers for yield and yield contributing traits in rice

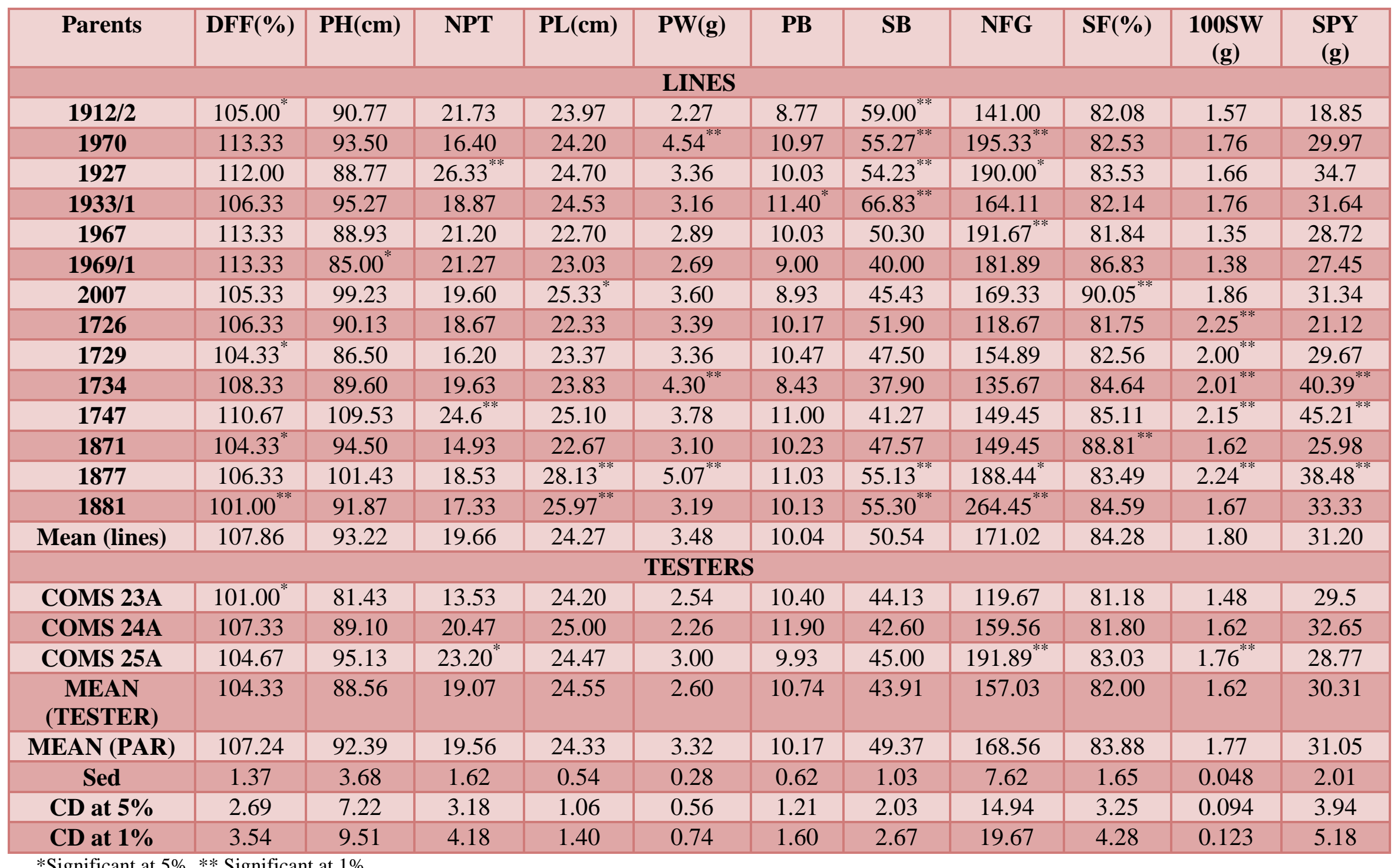

*Significant at $5 \%$,** Significant at $1 \%$ 
Table.4 General combining ability effects of parents for yield and yield contributing traits in rice

\begin{tabular}{|c|c|c|c|c|c|c|c|c|c|c|c|}
\hline Parents & $\operatorname{DFF}(\%)$ & $\mathbf{P H}(\mathbf{c m})$ & NPT & PL(cm) & $\mathbf{P W}(\mathbf{g})$ & PB & SB & NFG & SF(\%) & $\begin{array}{c}\text { 100SW } \\
(\mathrm{g})\end{array}$ & $\begin{array}{c}\text { SPY } \\
(\mathrm{g})\end{array}$ \\
\hline \multicolumn{12}{|c|}{ LINES } \\
\hline $1912 / 2$ & $6.39^{* *}$ & 0.44 & $2.87^{* *}$ & $0.91^{\text {** }}$ & $-0.26^{*}$ & 0.20 & $5.80^{* *}$ & $-13.65^{* *}$ & 0.48 & $-0.04^{*}$ & $-1.79^{*}$ \\
\hline 1970 & $6.72^{* *}$ & 2.77 & -0.23 & $0.80^{* *}$ & 0.06 & $1.29^{* *}$ & $7.26^{* *}$ & $-8.74^{*}$ & $-1.62^{*}$ & $-0.04^{*}$ & -1.21 \\
\hline 1927 & $4.83^{* *}$ & 1.39 & $-5.02^{* *}$ & $1.20^{* *}$ & $0.76^{* *}$ & $0.95^{* *}$ & $11.66^{* *}$ & $37.79^{* *}$ & -0.34 & $-0.15^{* *}$ & $5.58^{* *}$ \\
\hline $1933 / 1$ & $-1.17^{*}$ & 1.19 & $2.32^{* *}$ & $0.47^{*}$ & $-0.24^{*}$ & 0.13 & -0.24 & -0.27 & -0.45 & $-0.07^{* *}$ & $4.60^{* *}$ \\
\hline 1967 & $2.17^{* *}$ & 0.07 & $3.65^{* *}$ & 0.2 & $-0.30^{* *}$ & -0.53 & -0.18 & 0.24 & 0.66 & $-0.18^{* *}$ & $6.70^{* * *}$ \\
\hline $1969 / 1$ & 0.72 & -2.17 & $2.27^{* *}$ & $-0.48^{*}$ & -0.07 & 0.13 & $4.11^{* *}$ & $27.92^{* * *}$ & 0.02 & $-0.19^{* *}$ & $7.53^{* *}$ \\
\hline 2007 & $1.72^{\text {** }}$ & $4.28^{\text {** }}$ & $6.45^{* *}$ & 0.46 & -0.07 & $-0.78^{* *}$ & $-1.21^{*}$ & $-7.21^{*}$ & 0.1 & -0.00 & 0.20 \\
\hline 1726 & 0.61 & -0.42 & $-4.15^{* *}$ & -0.12 & -0.22 & 0.11 & $-1.29^{* *}$ & $-19.70^{* *}$ & -0.04 & $0.21^{* *}$ & $2.08^{* *}$ \\
\hline 1729 & $-5.94^{* *}$ & $-5.91^{* *}$ & -1.10 & $-0.57^{*}$ & 0.01 & 0.24 & $-7.32^{* *}$ & -2.94 & -0.85 & $0.08^{* *}$ & $-8.36^{* *}$ \\
\hline 1734 & -0.94 & $-4.39^{* *}$ & $-3.15^{* *}$ & $-0.79^{* *}$ & $0.34^{* * *}$ & 0.13 & $-4.38^{* *}$ & $17.52^{* *}$ & -0.08 & $0.11^{* *}$ & $3.02^{* *}$ \\
\hline 1747 & 0.39 & 2.68 & 1.03 & $-0.83^{* *}$ & 0.01 & -0.20 & $4.57^{* *}$ & $-7.19^{*}$ & 0.5 & $0.12^{* *}$ & 0.84 \\
\hline 1871 & $-3.83^{* * *}$ & $-3.55^{*}$ & $-3.28^{* *}$ & $-1.73^{* *}$ & 0.04 & -0.33 & $-8.19^{* *}$ & -6.30 & 0.28 & 0.03 & $-12.16^{* *}$ \\
\hline 1877 & $-8.39^{* * *}$ & $3.85^{*}$ & 0.27 & $1.07^{* *}$ & $0.49^{* *}$ & -0.38 & $-2.54^{* *}$ & -5.33 & $1.62 *$ & $0.20^{* *}$ & $3.69^{* *}$ \\
\hline 1881 & $-3.28^{* *}$ & -0.22 & $-1.92^{* *}$ & $-0.58^{*}$ & $-0.55^{* *}$ & $-0.96^{* * *}$ & $-8.05^{* *}$ & $-12.14^{* *}$ & -0.28 & $-0.06^{* *}$ & $-10.70^{* *}$ \\
\hline SE & 0.57 & 1.56 & 0.63 & 0.23 & 0.11 & 0.277 & 0.47 & 3.40 & 0.719 & 0.0175 & 0.79 \\
\hline \multicolumn{12}{|c|}{ TESTERS } \\
\hline COMS 23A & $-0.54^{*}$ & -0.78 & $-2.14^{* *}$ & $-0.33^{* *}$ & $0.52^{* *}$ & $0.35^{* *}$ & $1.84^{* *}$ & $15.18^{* *}$ & $-0.79^{*}$ & $-0.03^{* *}$ & $-1.10^{* *}$ \\
\hline COMS 24A & -0.49 & 1 & $1.29^{* *}$ & 0.21 & -0.07 & -0.06 & $0.60^{* *}$ & 0.83 & $1.40^{* *}$ & $0.06^{* *}$ & $2.79^{* *}$ \\
\hline COMS 25A & $1.03^{* *}$ & -0.22 & $0.85^{* *}$ & 0.12 & $-0.45^{* *}$ & $-0.29^{*}$ & $-2.43^{* *}$ & $-16.01^{* *}$ & -0.61 & $-0.03^{* *}$ & $-1.69^{* *}$ \\
\hline SE & 0.26 & 0.72 & 0.29 & 0.108 & 0.0519 & 0.128 & 0.22 & 1.57 & 0.33 & 0.0081 & 0.34 \\
\hline
\end{tabular}


Table.5 Mean of forty-two hybrids for yield and yield contributing traits in rice

\begin{tabular}{|c|c|c|c|c|c|c|c|c|c|c|c|}
\hline Hybrids & $\operatorname{DFF}(\%)$ & $\mathbf{P H}(\mathbf{c m})$ & NPT & PL(cm) & $\mathbf{P W}(\mathbf{g})$ & PB & SB & NFG & $\mathrm{SF}(\%)$ & $100 S W(g)$ & SPY $(g)$ \\
\hline COMS $23 A \times 1912 / 2$ & 107.00 & 91.87 & 24.13 & 24.83 & $4.80^{* *}$ & $12.40^{* *}$ & $60.83^{* *}$ & $187.67^{* *}$ & 83.39 & 1.76 & 35.65 \\
\hline COMS 23A×1970 & 107.33 & 97.30 & 22.23 & $26.07^{*}$ & $5.24^{* *}$ & $12.20^{*}$ & $56.67^{* *}$ & $194.47^{* *}$ & 81.80 & 1.88 & $49.46^{* *}$ \\
\hline COMS $23 A \times 1927$ & 104.33 & 95.30 & 17.13 & 24.70 & $4.17^{*}$ & 11.40 & $55.4^{* *}$ & $172.40^{* *}$ & 77.49 & 1.68 & $49.30^{* *}$ \\
\hline COMS 23A×1933/1 & 103.00 & 101.57 & 28.20 & 25.83 & 3.78 & 11.33 & $60.27^{* *}$ & $182.13^{* *}$ & 81.57 & 1.79 & $55.56^{* *}$ \\
\hline COMS 23A ×1967 & 103.00 & 91.43 & 21.93 & 24.20 & 3.26 & 10.53 & 48.33 & 143.80 & 80.29 & 1.62 & 38.29 \\
\hline COMS 23A×1969/1 & 102.33 & 88.67 & 22.33 & 24.17 & 3.17 & 11.53 & $51.07^{* *}$ & $167.87^{*}$ & 83.07 & 1.61 & 44.83 \\
\hline COMS 23A×2007 & 103.33 & 93.23 & 21.20 & 24.23 & 3.18 & 9.47 & 42.50 & 127.00 & 78.89 & 1.80 & 38.38 \\
\hline COMS 23A ×1726 & 102.67 & 91.53 & 13.40 & 24.20 & 3.57 & 11.07 & 46.77 & 138.07 & 78.54 & $2.33^{* *}$ & 31.95 \\
\hline COMS 23A $\times 1729$ & $97.33^{*}$ & $86.43^{*}$ & 17.53 & 24.20 & $4.23^{* *}$ & 11.20 & 40.97 & 145.07 & 78.72 & $2.14^{* *}$ & 31.16 \\
\hline COMS 23A×1734 & $97.67^{*}$ & 94.33 & 16.40 & 24.77 & $4.78^{* *}$ & 11.27 & $50.13^{* *}$ & $192.07^{* *}$ & 77.86 & 1.96 & $46.26^{*}$ \\
\hline COMS 23A ×1747 & 102.33 & 97.63 & 19.33 & 24.17 & 3.91 & 10.87 & $56.13^{* *}$ & 149.13 & 79.85 & $2.12^{* *}$ & $47.06^{* *}$ \\
\hline COMS 23A ×1871 & $86.33^{* *}$ & 91.83 & 18.93 & 23.4 & $4.11^{*}$ & 9.73 & 32.67 & $166.73^{*}$ & 80.28 & 2.03 & 25.02 \\
\hline COMS 23A×1877 & $89.67^{* *}$ & 87.67 & 18.93 & 25.5 & $4.62^{* *}$ & 10.4 & 43.93 & 147.6 & 82.26 & $2.13^{* *}$ & 45.41 \\
\hline COMS $23 A \times 1881$ & $94.67^{* *}$ & 97.17 & 17.47 & 24.77 & 2.7 & 10.87 & 37.87 & $174.13^{* *}$ & 79.82 & 1.98 & 30.55 \\
\hline COMS 24A×1912/2 & 107.00 & 96.77 & $26.47^{* *}$ & $26.20^{*}$ & 2.41 & 10.00 & $50.70^{* *}$ & 109.67 & 83.90 & 2.03 & $50.43^{* *}$ \\
\hline COMS 24A×1970 & 109.00 & 97.00 & 24.87 & $26.23^{*}$ & 2.83 & $12.07^{*}$ & $54.83^{* *}$ & 120.13 & 79.34 & 1.92 & 42.36 \\
\hline COMS 24A×1927 & 105.33 & 98.07 & 18.73 & $26.30^{*}$ & $4.04^{*}$ & 11.60 & $57.7^{* *}$ & $189.33^{* *}$ & 82.97 & 1.98 & $58.48^{* *}$ \\
\hline COMS $24 A \times 1933 / 1$ & 108.00 & 94.13 & 24.73 & 25.20 & 3.47 & 10.80 & 44.33 & 137.00 & 79.84 & 1.99 & $47.80^{* *}$ \\
\hline COMS 24A×1967 & 102.00 & 92.80 & $27.07^{* *}$ & 25.77 & 3.14 & 9.73 & 45.20 & $163.40^{*}$ & 81.98 & 1.79 & $46.11^{*}$ \\
\hline COMS 24A×1969/1 & 98.67 & 92.97 & $28.27^{* *}$ & 24.73 & 3.89 & 10.60 & 53.50 & $215.20^{* *}$ & 83.73 & 1.92 & $56.75^{* *}$ \\
\hline COMS 24A×2007 & 102.00 & 100.8 & $28.33^{* *}$ & $26.30^{*}$ & 3.98 & 10.67 & 51.07 & $173.67^{* *}$ & 83.09 & 2.01 & $51.27^{* *}$ \\
\hline COMS 24A×1726 & 98.67 & 93.50 & 16.67 & 24.17 & 2.70 & 10.00 & 43.50 & 109.07 & 82.03 & 2.00 & 31.04 \\
\hline COMS 24A ×1729 & $86.00^{* *}$ & $86.53^{*}$ & 22.93 & 23.73 & 2.94 & 11.00 & 43.30 & 143.93 & 82.46 & 1.97 & 28.56 \\
\hline COMS 24A×1734 & $97.00^{* *}$ & 94.1 & 22.07 & 24.3 & 3.01 & 10.07 & 39.43 & 126.2 & 82.95 & $2.17^{* *}$ & $49.50^{* *}$ \\
\hline COMS 24A×1747 & 100.33 & 97.1 & 19.93 & 24.2 & 3.54 & 10.93 & $52.53^{* *}$ & 154.47 & 83.15 & 1.99 & 35.80 \\
\hline Hybrids & $\operatorname{DFF}(\%)$ & $\mathbf{P H}(\mathbf{c m})$ & NPT & PL(cm) & $\mathbf{P W}(\mathbf{g})$ & PB & SB & NFG & $\mathrm{SF}(\%)$ & $100 S W(g)$ & SPY $(g)$ \\
\hline COMS 24A×1871 & 101.00 & 91.33 & 19.2 & 23.67 & 3.81 & 11.4 & 44.9 & $163.33^{*}$ & 82.14 & 1.87 & 36.35 \\
\hline COMS 24A×1877 & $85.67^{* *}$ & 102.33 & 24.05 & $26.23^{*}$ & $4.07^{*}$ & 10.33 & 45.57 & 142.7 & $84.41^{*}$ & $2.36^{* *}$ & $51.76^{* *}$ \\
\hline COMS 24A×1881 & 101.00 & 93.57 & 23.93 & 25.6 & 3.38 & 9.33 & 39.63 & 139 & 82.58 & 2.02 & 37.09 \\
\hline COMS 25A ×1912/2 & 107.00 & 94.9 & 24.27 & $26.63^{* *}$ & 2.33 & 10.20 & 46.83 & 106.47 & 77.35 & 1.92 & 33.75 \\
\hline COMS 25A×1970 & 105.67 & 96.20 & 18.47 & 25.03 & 2.44 & 11.60 & $51.23^{* *}$ & 103.93 & 77.18 & 1.91 & 29.74 \\
\hline
\end{tabular}




\begin{tabular}{|c|c|c|c|c|c|c|c|c|c|c|c|}
\hline COMS 25A ×1927 & 106.67 & 93.00 & 15.33 & $27.53^{* *}$ & $4.40^{* *}$ & 11.87 & $62.83^{* *}$ & $196.40^{* *}$ & 81.71 & 1.72 & 34.15 \\
\hline COMS 25A ×1933/1 & $87.33^{* *}$ & 90.07 & 20.27 & 25.30 & 2.38 & 10.27 & 35.63 & 124.81 & 80.43 & 1.83 & 35.65 \\
\hline COMS 25A ×1967 & 103.33 & 98.17 & $28.2^{* *}$ & 25.57 & 3.02 & 10.13 & 46.90 & 138.27 & 82.92 & 1.89 & $60.90^{*}$ \\
\hline COMS $25 A \times 1969 / 1$ & 103.00 & 94.07 & 22.47 & 24.60 & 3.08 & 10.27 & 48.73 & 145.47 & 76.47 & 1.73 & $46.21^{*}$ \\
\hline COMS 25A×2007 & 101.67 & 101.00 & $36.07^{* *}$ & 25.77 & 2.95 & 9.53 & 43.77 & 122.47 & 81.52 & 2.01 & 36.15 \\
\hline COMS 25A ×1726 & 102.33 & 95.90 & 23.73 & $26.20^{*}$ & 3.41 & 11.27 & 46.83 & 138.53 & 82.53 & $2.15^{* *}$ & $68.44^{* *}$ \\
\hline COMS 25A×1729 & 100.67 & 91.50 & 22.48 & 25.30 & 3.21 & 10.53 & 34.73 & 146.93 & 79.48 & 1.95 & 40.39 \\
\hline COMS 25A ×1734 & 104.33 & $80.6^{* *}$ & 18.33 & 23.5 & 3.55 & 11.07 & 38.27 & $179.07^{* *}$ & 82.13 & 2.02 & 38.49 \\
\hline COMS 25A ×1747 & 100.33 & 95.5 & $30.07^{* *}$ & 24.07 & 2.9 & 9.6 & 46 & 119.6 & 81.72 & $2.08^{* * *}$ & 44.84 \\
\hline COMS 25A ×1871 & 103.00 & 88.4 & 18.27 & 22.67 & 2.54 & 9.87 & 38.83 & 95.8 & 81.61 & $2.04^{*}$ & 27.34 \\
\hline COMS 25A ×1877 & 101.33 & 103.77 & 24.07 & $26.4^{* *}$ & 3.11 & 10.13 & 43.83 & 138.47 & 81.38 & 1.94 & 39.10 \\
\hline COMS 25A ×1881 & $96.33^{* *}$ & 90.8 & 19.1 & 22.83 & 2.6 & 8.93 & 39.3 & 95.2 & 79.95 & 1.64 & 25.46 \\
\hline $\begin{array}{c}\text { Mean } \\
\text { (hybrids) }\end{array}$ & 100.61 & 94.07 & 22.08 & 24.98 & 3.44 & 10.67 & 46.99 & 148.25 & 81.07 & 1.94 & 41.73 \\
\hline SEd & 1.37 & 3.68 & 1.62 & 0.54 & 0.28 & 0.62 & 1.03 & 7.62 & 1.65 & 0.048 & 2.01 \\
\hline CD at $5 \%$ & 2.69 & 7.22 & 3.18 & 1.06 & 0.56 & 1.21 & 2.03 & 14.94 & 3.24 & 0.094 & 3.94 \\
\hline CD at $1 \%$ & 3.54 & 9.51 & 4.18 & 1.40 & 0.74 & 1.60 & 2.67 & 19.67 & 4.27 & 0.123 & 5.18 \\
\hline
\end{tabular}

*Significant at $5 \%$, ** Significant at $1 \%$

Table.6 Specific combining ability effects of forty-two hybrids for yield and yield contributing traits in rice

\begin{tabular}{|c|c|c|c|c|c|c|c|c|c|c|c|}
\hline Hybrids & $\operatorname{DFF}(\%)$ & PH(cm) & NPT & PL(cm) & $\mathbf{P W}(\mathrm{g})$ & PB & SB & NFG & SF $(\%)$ & $100 S W(g)$ & SPY $(g)$ \\
\hline COMS 23A $\times 1912 / 2$ & 0.54 & -1.86 & 1.32 & -0.72 & $1.10^{* *}$ & $1.18^{*}$ & $6.21^{* *}$ & $37.88^{* *}$ & $2.63^{*}$ & $-0.12^{* * *}$ & $-3.19^{*}$ \\
\hline COMS $23 \mathrm{~A} \times 1970$ & 0.54 & 1.25 & 2.52 & 0.62 & $1.21^{* *}$ & -0.11 & 0.59 & $39.77^{* *}$ & $3.15^{*}$ & 0.00 & $10.04^{* *}$ \\
\hline COMS 23A×1927 & -0.57 & 0.63 & $2.21^{*}$ & $-1.15^{* *}$ & $-0.55^{* *}$ & -0.57 & $-5.08^{* *}$ & $-28.83^{* *}$ & -2.44 & $-0.08^{* *}$ & $3.09^{*}$ \\
\hline COMS 23A×1933/1 & $4.10^{* *}$ & $7.10^{*}$ & $5.94^{* *}$ & 0.72 & 0.05 & 0.18 & $11.69^{* *}$ & $18.97^{* *}$ & 1.75 & -0.05 & $10.33^{* *}$ \\
\hline COMS 23A×1967 & 0.76 & -1.92 & -1.66 & -0.65 & $-0.41^{*}$ & 0.05 & -0.31 & $-19.87^{* *}$ & -0.65 & $-0.12^{* *}$ & $-9.04^{* *}$ \\
\hline COMS 23A×1969/1 & 1.54 & -2.45 & 0.12 & 0.00 & $-0.73^{* *}$ & 0.38 & $-1.87^{*}$ & $-23.50^{* *}$ & $2.77^{*}$ & $-0.11^{* *}$ & $-3.34^{*}$ \\
\hline COMS 23A×2007 & 1.54 & -4.33 & $-5.19^{* *}$ & $-0.87^{*}$ & $-0.71^{* *}$ & -0.77 & $-5.11^{* *}$ & $-29.2^{* *}$ & -1.49 & $-0.11^{* *}$ & -2.45 \\
\hline COMS 23A $\times 1726$ & 1.98 * & -1.33 & $-2.39^{*}$ & -0.32 & -0.18 & -0.06 & -0.77 & -5.67 & -1.7 & $0.20^{* *}$ & $-10.76^{* *}$ \\
\hline COMS 23A $\times 1729$ & $3.21^{* *}$ & -0.94 & -1.31 & 0.12 & 0.25 & -0.06 & -0.54 & $-15.43^{*}$ & -0.71 & $0.15^{* *}$ & -1.11 \\
\hline COMS 23A $\times 1734$ & -1.46 & $5.44^{*}$ & -0.39 & $0.91^{*}$ & $0.48^{*}$ & 0.12 & $5.69^{* *}$ & 11.10 & -2.33 & $-0.06^{*}$ & 2.61 \\
\hline COMS 23A×1747 & 1.87 & 1.67 & -1.63 & 0.35 & -0.06 & 0.05 & $2.74^{* *}$ & -7.12 & -0.93 & $0.09^{* *}$ & $5.60^{* *}$ \\
\hline COMS 23A×1871 & $-9.90^{* *}$ & 2.10 & $2.28^{*}$ & 0.49 & 0.10 & -0.95 & $-7.97^{* *}$ & 9.59 & -0.27 & $0.08^{*}$ & $-3.45^{*}$ \\
\hline
\end{tabular}




\begin{tabular}{|c|c|c|c|c|c|c|c|c|c|c|c|}
\hline COMS 23A $\times 1877$ & $-2.02^{*}$ & -9.47 & -1.27 & -0.21 & 0.17 & -0.24 & $-2.35^{* *}$ & -10.15 & 0.37 & 0.02 & 1.09 \\
\hline COMS 23A×1881 & $-2.13^{*}$ & 4.11 & -0.56 & 0.70 & $-0.72^{* *}$ & 0.80 & $-2.90^{* *}$ & $22.84^{* * *}$ & -0.17 & $0.13^{* *}$ & 0.62 \\
\hline COMS 24A×1912/2 & 0.49 & 1.25 & 0.22 & 0.10 & $-0.70^{* *}$ & -0.81 & $-2.69^{* *}$ & $-25.76^{* *}$ & 0.95 & $0.07^{*}$ & $7.69^{* *}$ \\
\hline COMS 24A×1970 & $2.16^{*}$ & -0.84 & 1.72 & 0.25 & $-0.60^{* *}$ & 0.17 & -0.01 & $-20.20^{* *}$ & -1.50 & -0.04 & -0.95 \\
\hline COMS 24A×1927 & 0.38 & 1.61 & 0.38 & -0.09 & -0.09 & 0.04 & -1.54 & 2.46 & 0.84 & $0.13^{* *}$ & $8.38^{* *}$ \\
\hline COMS 24A×1933/1 & $9.05^{* * *}$ & -2.13 & -0.96 & -0.45 & 0.33 & 0.06 & $-3.01^{* *}$ & $-11.81^{*}$ & -2.18 & $0.07^{*}$ & -1.33 \\
\hline COMS 24A×1967 & -0.29 & -2.34 & 0.04 & 0.38 & 0.07 & -0.34 & $-2.21^{* *}$ & $14.09^{*}$ & -1.16 & -0.04 & $-5.11^{* *}$ \\
\hline COMS 24A×1969/1 & $-2.17^{*}$ & 0.06 & $2.62^{*}$ & 0.02 & $0.58^{* *}$ & -0.14 & $1.80^{*}$ & $38.20^{* *}$ & 1.24 & $0.11^{* *}$ & $4.70^{* *}$ \\
\hline COMS 24A×2007 & 0.16 & 1.45 & -1.49 & 0.66 & $0.68^{* *}$ & 0.84 & $4.69^{* *}$ & $31.80^{* *}$ & 0.52 & 0.01 & $6.55^{* *}$ \\
\hline COMS 24A×1726 & $-2.06^{*}$ & -1.15 & $-2.56^{*}$ & $-0.90^{*}$ & $-0.45^{*}$ & -0.72 & $-2.80^{* *}$ & $-20.31^{* *}$ & -0.41 & $-0.22^{* *}$ & $-15.56^{* *}$ \\
\hline COMS 24A $\times 1729$ & $-8.17^{* *}$ & -2.63 & 0.66 & $-0.89^{*}$ & $-0.45^{*}$ & 0.15 & $3.03^{* *}$ & -2.20 & 0.84 & $-0.10^{* *}$ & $-7.60^{* *}$ \\
\hline COMS 24A × 1734 & $-2.17^{*}$ & 3.42 & 1.84 & -0.10 & $-0.70^{* *}$ & -0.67 & $-3.78^{* *}$ & $-40.40^{* *}$ & 0.57 & $0.06^{*}$ & 1.96 \\
\hline COMS 24A×1747 & -0.17 & -0.65 & $-4.47^{* *}$ & -0.15 & 0.16 & 0.53 & 0.38 & $12.57^{*}$ & 0.18 & $-0.13^{* *}$ & $-9.55^{* *}$ \\
\hline COMS 24A×1871 & $4.71^{* *}$ & -0.19 & -0.89 & 0.21 & $0.40^{*}$ & 1.13 & $5.50^{* *}$ & $20.55^{* *}$ & -0.61 & $-0.16^{* *}$ & $4.00^{* *}$ \\
\hline COMS 24A×1877 & $-6.06^{* *}$ & 3.41 & 0.14 & -0.02 & 0.21 & 0.10 & 0.52 & -1.05 & 0.33 & $0.16^{* * *}$ & $3.55^{*}$ \\
\hline COMS 24A×1881 & $4.16^{* *}$ & -1.28 & $2.48^{*}$ & $0.99^{*}$ & $0.56^{* *}$ & -0.32 & 0.10 & 2.06 & 0.39 & $0.08^{* *}$ & $3.27^{*}$ \\
\hline COMS 25A ×1912/2 & -1.03 & 0.61 & -1.54 & 0.62 & $-0.40^{*}$ & -0.37 & $-3.52^{* *}$ & $-12.12^{*}$ & $-3.58^{* *}$ & 0.04 & $-4.50^{* *}$ \\
\hline COMS 25A $\times 1970$ & $-2.70^{* *}$ & -0.41 & $-4.24^{* *}$ & $-0.87^{*}$ & $-0.61^{* *}$ & -0.06 & -0.58 & $-19.57^{* *}$ & -1.65 & 0.04 & $-9.09^{* *}$ \\
\hline COMS 25A ×1927 & 0.19 & -2.24 & $-2.59^{*}$ & $1.23^{* *}$ & $0.65^{* *}$ & 0.54 & $6.62^{* *}$ & $26.37^{* *}$ & 1.59 & -0.04 & $-11.47^{* *}$ \\
\hline COMS 25A×1933/1 & $-13.14^{* * *}$ & -4.97 & $-4.99^{* *}$ & -0.27 & -0.38 & -0.24 & $-8.68^{* *}$ & -7.16 & 0.43 & -0.01 & $-9.00^{* *}$ \\
\hline COMS 25A $\times 1967$ & -0.48 & 4.25 & 1.61 & 0.27 & 0.33 & 0.29 & 2.52 & 5.79 & 1.80 & $0.15^{* *}$ & $14.16^{* * *}$ \\
\hline COMS 25A×1969/1 & 0.63 & 2.39 & $-2.74^{*}$ & -0.02 & 0.15 & -0.24 & 0.07 & $-14.70^{*}$ & $-4.01^{* *}$ & 0.01 & -1.36 \\
\hline COMS 25A×2007 & -1.70 & 2.88 & $6.68^{* * *}$ & 0.21 & 0.03 & -0.06 & 0.42 & -2.57 & 0.97 & $0.10^{* *}$ & $-4.09^{* * *}$ \\
\hline COMS 25A×1726 & 0.08 & 2.48 & $4.95^{* *}$ & $1.22^{* *}$ & $0.63^{* *}$ & 0.78 & $3.57^{* *}$ & $25.99^{* *}$ & 2.11 & 0.02 & $26.32^{* *}$ \\
\hline COMS 25A $\times 1729$ & $4.97^{* *}$ & 3.56 & 0.65 & 0.77 & 0.20 & -0.09 & $-2.50^{* *}$ & $17.63^{* *}$ & -0.13 & -0.04 & $8.71^{* *}$ \\
\hline COMS 25A×1734 & $3.63^{* *}$ & $-8.86^{* *}$ & -1.45 & $-0.81^{*}$ & 0.22 & 0.56 & $-1.91^{*}$ & $29.30^{* * *}$ & 1.76 & -0.00 & $-4.57^{* *}$ \\
\hline COMS 25A $\times 1747$ & -1.70 & -1.02 & $6.10 * *$ & -0.2 & -0.10 & -0.57 & $-3.12^{* *}$ & -5.46 & 0.76 & 0.04 & $3.96^{* *}$ \\
\hline COMS 25A ×1871 & $5.19^{* *}$ & -1.90 & -1.39 & -0.7 & -0.49 & -0.17 & $2.47^{* *}$ & $-30.15^{* *}$ & 0.87 & $0.09^{* *}$ & -0.54 \\
\hline COMS 25A ×1877 & $8.08^{* *}$ & $6.06^{*}$ & 0.86 & 0.23 & -0.37 & 0.14 & $1.82^{*}$ & 11.56 & -0.69 & $-0.18^{* *}$ & $-4.64^{* *}$ \\
\hline COMS 25A ×1881 & $-2.03^{*}$ & -2.82 & -1.92 & $-1.69^{* *}$ & 0.16 & -0.49 & $2.80^{* *}$ & $-24.90^{* *}$ & -0.22 & $-0.21^{* *}$ & $-3.88^{* *}$ \\
\hline $\begin{array}{c}\text { SE } \\
\text { (hybrids) }\end{array}$ & 0.99 & 2.69 & 1.0905 & 0.405 & 0.1943 & 0.4801 & 0.811 & 5.896 & 1.25 & 0.030 & 1.36 \\
\hline
\end{tabular}


Heterosis, though defined as increased or decreased vigor of $F_{1}$ hybrids over either parent for yield and different yield attributing traits, it has practical utility only if the gain is on the positive side for the major trait, plant yield. Thus, heterosis for grain yield is a pivotal factor considered for developing hybrids with phenomenal performance. In the current study, hybrids were grouped on the basis of their duration viz., Early (five hybrids), Mid early (six hybrids) and medium duration hybrids (31 hybrids) and standard heterosis worked out against the varietal and hybrid checks of respective duration for drawing meaningful conclusions.

Early hybrids were compared with $\mathrm{CO}$ 51(varietal check) and CORH3 (hybrid check) for their yield superiority. The standard heterosis over varietal check $(31.7 \mathrm{~g})$ ranged from -21.49 to $62.43 \%$ and hybrid check $(32.32 \mathrm{~g})$ ranged from -22.60 to $60.14 \%$. For both the checks, only two hybrids COMS 23A $\times 1877\left(42.53^{* *} \%, 40.51^{* *} \%\right)$ and COMS $24 \mathrm{~A} \times 1877\left(62.43^{* *} \%, 60.14^{* *} \%\right)$ exhibited highly significant heterosis. Mid early hybrids were evaluated with ADT 39 (varietal check) and US 312 (National hybrid check). Standard heterosis ranged from -29.31 to $37.47 \%$ for ADT 39 (36.01g). For US312 (40.44g), standard heterosis ranged from -37.05 to $22.41 \%$. Two crosses namely COMS $23 \mathrm{~A} \times$ $1734\left(28.46^{* *} \%, 14.38^{* *} \%\right)$ and COMS $24 \times$ $1734\left(37.47^{* *} \%, \quad 22.41^{* *} \%\right)$ had highly significant heterosis over both checks. Medium duration hybrids were assessed for their superiority with CO 50 (varietal check) and HRI 174 (National hybrid check). The standard heterosis ranged from to -40.83 to $48.13 \%$ for CO $50(46.20 \mathrm{~g})$ and for HRI 174 $(44.35 \mathrm{~g})$ the range was from -38.36 to $54.32 \%$. Against both the checks, seven crosses exhibited significant heterosis viz., COMS24 Ax 1912/2(9.15*,13.71**)COMS $24 \mathrm{~A} \times 2007\left(10.98^{*} \%, 15.62^{* *} \%\right)$, COMS 23A $\times 1933 / 1 \quad\left(20.27^{* *} \%, 25.29^{* *} \%\right)$,
COMS24A $\times 1969 / 1\left(22.84^{* *} \%, 27.98^{* *} \%\right)$, COMS 24A $\times 1927\left(26.57^{* *} \%, 31.86^{* *} \%\right)$, COMS 25A x $1967\left(31.81^{* *} \%, 37.32^{* *} \%\right)$ and COMS 25A × $1726\left(48.13^{* *} \%, 54.32^{* *} \%\right)$. Thippeswamy et al., (2016) had evaluated sixty-five hybrids with two standard checks JGL18047 and MTU1010 and reported three hybrids having significant standard heterosis over both checks. Top two heterotic combinations identified for grain yield per plant by Thorat et al., (2017)were IR58025A $x$ NPQ-49 and RTN12A x NPQ-49.Twentyone hybrids displayed significant positive standard heterosis for grain yield over hybrid check (KRH2) and varietal check MTU (1010) in the experiments of Parimala et al., (2018).

When we consider significant mean performance, sca effects, gca effects of parents and standard heterosis for grain yield, eight hybrids viz., COMS 23A × 1933/1, COMS 24A $\times 1912 / 2$, COMS 24A $\times 1927$, COMS 24A $\times 1969 / 1$, COMS 24A $\times 2007$, COMS 24A $\times 1877$, COMS $25 \mathrm{~A} \times 1967$ and COMS $25 \mathrm{~A} \times 1726$ emanated as heterotic for single plant yield.. Tiwari et al., (2011) identified six hybrids out of 60 combinations as promising based on above $50 \%$ heterosis for grain yield and other yield components as well as significant sca effects for major components.

The identified hybrids in our study had parents with low/high, high/low, high/moderate and high/high general combining effects. Crosses COMS 23A $\times$ 1933/1, COMS 25A× 1967 and COMS 25A $\times$ 1726 had parents with low/high $g c a$ effects. Cross COMS 24A × 1912/2 had high/low gca effect. These crosses show the presence of dominant $x$ additive type of gene interaction. Hybrid COMS 24A × 2007 involved high/moderate parental combination. Hybrids COMS 24A ×1927, COMS 24A × 1969/1 and COMS 24A× 1877 had high/high general 
combining parents. These combinations indicate the presence of additive $x$ additive gene interaction. From the above results we can conclude that there is no particular relationship between positive significant $s c a$ effects of hybrids and their parental parental gca effects. The two heterotic hybrids reported by Thorat et al., (2017) involved parents with average $\times$ good combining ability. Singh et al., (2019) also reported eight hybrids possessing positive significant sca effects with no particular relation for respective parental gca effects. It has also been emphasized that superior parents do not always produce superior heterotic combinations, moreover, parents of elite heterotic combinations are always developed from different heterotic groups (Zeng et al., 2007).

Hybrid rice exploits mostly the Wild abortive (WA) Cytoplasmic - genic Male Sterility (CMS) for the development of hybrids and seed multiplication.The results witnessed as high per se performance and significant specific combining ability effects in hybrids offer scope for commercialization after large scale seed production and testing provided the standard heterosis is appreciable and the flowering synchrony between parents could be achieved during seed production.

From the above results and discussion, we can infer that the expression of various yield and yield attributing characters in current study is controlled by non-additive gene action. Parents 1927,1877 and COMS 24A can be inferred as best combiners for five to six traits and can be used in future breeding programmes. The $\mathrm{F}_{1} \mathrm{~s}$ of eightcross combinations were adjudged as superior hybrids based on mean and combining ability parameters. All hybrids had good flowering synchrony between respective parents (less than nine days) that can be exploited in the near future for re-evaluation and large-scale testing. In an earlier study by Jeevanapriya (2019), it was observed that the lines of best hybrids fall indifferent cluster in molecular diversity analysis.

\section{References}

AICRIP progress report 2019, Vol-1 varietal improvement

Cheng, S.H., J.Y. Zhuang, Y.Y. Fan, J.H. Du, and L.Y. Cao. 2007. "Progress in research and development on hybrid rice: a super-domesticate in China." Annals of botany 100 (5):959-966.

Devi, B., and G. Lal. 2015. "Combining ability analysis for yield \& yield components in rice (Oryza sativa L.)." Electronic Journal of Plant Breeding 6 (2):360-365.

Gramaje, L., J. Caguiat, J. Carampatana, and R. Millas. 2016. "Heterosis and combining ability of selected parent lines for hybrid rice (Oryza sativa L.) development in PhilRice hybrid rice breeding pool." Philippine Journal of Crop Science (Philippines) 216.

Hasan, M., M. Kulsum, E. Hossain, M.M. Hossain, M.M. Rahman, and N.M.F. Rahmat. 2015."Combining ability analysis for identifying elite parents for heterotic rice hybrids." Academia Journal of Agricultural Research 3 (5):70-75.

Hwa, C.M., and X.C. Yang. 2008. "Fixation of hybrid vigor in rice: opportunities and challenges." Euphytica 160 (3):287293.

Jeevanapriya, P. 2019 "Restorer Identification and Dissecting their Genetic diversity in Hybrid Rice" TamilNadu Agricultural University.

Kempthorne, O. 1957. "An introduction to genetic statistics."

Ni, X.L., T. Zhang, K.F. Jiang, L. Yang, Q.H. Yang, Y.J. Cao, C.Y. Wen, and J.K. Zheng. 2009. "Correlations between 
specific combining ability, heterosis and genetic distance in hybrid rice." $Y i$ Chuan Hereditas 31 (8):849-854.

Osama, E.B., H. El-Mowafi, and A. Abd ElHadi. 2017. "Genetic Analysis for $\mathrm{F}_{1}$ Rice Hybrids Developed Using Three Cytoplasmic Male Sterile Line System." International Journal of Agricultural and Environmental Sciences 2 (5):60.

Panse, V., and P. Sukhatme. 1985. "Statistical methods for agricultural research."Indian Council of Agricultural Research, New Delhi 8:308-318.

Parimala, K., D. Bhadru, and C.S. Raju. 2018. "Combining ability and heterosis studies for grain yield and its components in hybrid rice (Oryza sativa L.)." Electronic Journal of Plant Breeding 9 (1):244-255.

Singh, Y., P. Singh, V. Pathak, V. Misra, and A. Mall. 2019. "Combining ability and gene action for grain yield using Line $x$ Tester technique in aerobic rice." Plant Archives 19 (1):1135-1140.

Sudeepthi, K., D. Jyothula, and Y. Suneetha. 2018. "Heterosis and Combining Ability Studies for Yield and Yield Component Traits in Rice (Oryza sativa L.)." International Journal of Current
Microbiologyand Applied Sciences 7 (10):1205-1211.

Thippeswamy, S., Y.C. Mohan, B. Srinivas, D. Padmaja, Z. Samreen, and S. Laxman. 2016. "Development of high yielding rice hybrids through heterosis and combining ability analysis." The Ecoscan 10 (1\&2):227-231.

Thorat, B., R. Kunkerkar, and M. Chavan. 2017. "Heritability study in hybrid rice (Oryza sativa L.)." Journal of Pharmacognosy and Phytochemistry 6 (5):1450-1453.

Tiwari, D.,P. Pandey, S. Giri, and J. Dwivedi. 2011. "Prediction of gene action, heterosis and combining ability to identify superior rice hybrids." International Journal of Botany 7 (2):126-144.

Virmani, S., and I.B. Edwards. 1983."Current status and future prospects for breeding hybrid rice and wheat." Advances in Agronomy 36:145-214.

Zeng, M.Q., H.l. Ji, J. Y. Li, and J. Sansen. 2007. "Formation and Development on the Conception of Heterotic Group and Their Heterotic Pattern in Maize (Zea mays L.)[J]." Acta Agriculturae Boreali-Sinica 6.

\section{How to cite this article:}

Jayalakshmi' R., R. Saraswathi and Amudha, K. 2021. Investigations on Combining Ability and Heterosis in Hybrid Rice. Int.J.Curr.Microbiol.App.Sci. 10(02): 2838-2850. doi: https://doi.org/10.20546/ijcmas.2021.1002.315 